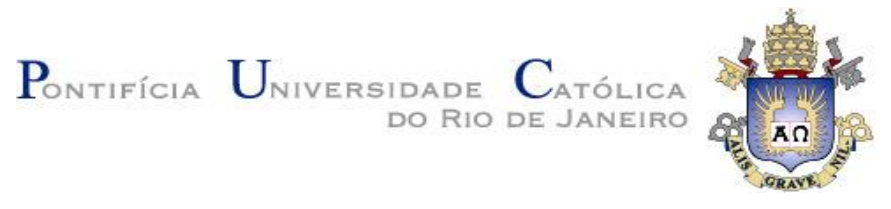

Marcelo Molina Silva

\begin{abstract}
Avaliação de Algoritmos de Detecção de Espaços Espectrais Brancos para Aplicações de Rádio Cognitivo
\end{abstract}

Dissertação apresentada como requisito parcial para obtenção do grau de Mestre pelo Programa de Pósgraduação em Engenharia Elétrica do Departamento de Engenharia Elétrica da PUC-Rio.

Orientador: Prof. Luiz Alencar Reis da Silva Mello

Rio de Janeiro

Março de 2014 


\section{Avaliação de Algoritmos de Detecção de Espaços Espectrais Brancos para Aplicações de Rádio Cognitivo}

Dissertação apresentada como requisito parcial para obtenção do grau de Mestre pelo Programa de Pósgraduação em Engenharia Elétrica do Departamento de Engenharia Elétrica da PUC-Rio.

Prof. Luiz Alencar Reis da Silva Mello

Orientador

Centro de Estudos em Telecomunicações - PUC-Rio

Prof. Carlos Vinicio Rodríguez Ron

Co-orientador

Centro de Estudos em Telecomunicações - PUC-Rio

Prof. Leni Joaquim de Matos Universidade Federal Fluminense - UFF

Prof. Marco Antonio Grivet Mattoso Maia Centro de Estudos em Telecomunicações - PUC-Rio

Prof. Pedro Vladimir Gonzalez Castellanos INMETRO

Prof. José Eugênio Leal Coordenador Setorial do Centro

Técnico Científico - PUC-Rio Rio de Janeiro, 28 de março de 2014 
Todos os direitos reservados. É proibida a reprodução total ou parcial do trabalho sem autorização da universidade, do autor e do orientador.

\section{Marcelo Molina Silva}

Graduou-se em Engenharia de Telecomunicações, na Universidad Católica Boliviana "San Pablo" UCB (La Paz Bolívia)

Ficha Catalográfica

Silva, Marcelo Molina

Avaliação de algoritmos de detecção de espaços espectrais brancos para aplicações de rádio cognitivo / Marcelo Molina Silva; orientador: Luiz Alencar Reis da Silva Mello.- 2014.

129 f. : il. (color.) ; $30 \mathrm{~cm}$

Dissertação (mestrado)-Pontifícia Universidade Católica do Rio de Janeiro, Departamento de Engenharia Elétrica, 2014.

Inclui bibliografia

1. Engenharia elétrica - Teses. 2. Rádio cognitivo. 3. Intervalos espectrais. 4. Algoritmos de detecção. 5. Complexidade computacional. I. Mello, Luiz Alencar Reis da Silva. II. Pontifícia Universidade Católica do Rio de Janeiro. Departamento de Engenharia Elétrica. III. Título. 
Aos meus queridos pais Andrés Molina e Rebeca Silva, aos meus queridos irmãos, sobrinhos e cunhados. 


\section{Agradecimentos}

Graças a Deus, acima de todas as coisas, esta é mais uma prova de sua presença em minha vida.

Ao Prof. Luiz Alencar Reis da Silva Mello, orientador deste trabalho, pela confiança e orientação ao longo deste projeto e, ainda mais, por sua amizade.

Ao Calos Vinicio Rodríguez Ron, co-orientador deste trabalho, e Pedro Vladimir Gonzalez Castellanos, por sua colaboração no desenvolvimento deste trabalho.

Aos meus pais, Andrés Molina e Rebeca Silva, por todo o amor, força e pelas palavras de apoio nos momentos mais difíceis, que me deram todos os dias. Foram, são e sempre serão meu exemplo a seguir.

Aos meus irmãos: Andrés, Alejandra e Fernanda por cada momento que estiveram ao meu lado e todo o amor, que eu não tenho palavras para dizer o quanto eu aprecio e admiro vocês.

Aos meus cunhados: Carla, Giovanni, Pablo e meus sobrinhos: Andres, Fernando, Ignácio e para o bebê (Sebastian) da minha irmã Alejandra que esperamos ansiosos, por todo apoio em todo este tempo.

A Gricel, Fabricio, Fatima e Sandra pela amizade incondicional, por estar cada momento a meu lado, por oferecer uma amizade tão valiosa, brigado por cada momento que vivi ao lado de vocês.

A Lucia e Luciana por abrir as portas de sua casa dando-me o carinho de uma família aqui no Rio de Janeiro.

A meus amigos do CETUC, especialmente a Jennifer, Junior e Romar além de serem meus companheiros de aula formamos uma bela amizade, Beatriz, Alex e Uwe, pela sua amizade e colaboração na elaboração deste trabalho, a meus amigos da Bolívia que também estudam no CETUC: Ariel, Carlos, Daniel, Diego, Mauricio, Teddy, pela sua amizade e apoio ao longo deste trabalho.

A meus amigos da Bolívia, que apesar da distância mantemos essa amizade, muito obrigado pelo apoio de cada um de vocês.

À Fundação Capes e a PUC-Rio pelo fomento da bolsa durante a realização deste trabalho. 


\section{Resumo}

Molina Silva, Marcelo; da Silva Mello, Luiz Alencar Reis. Avaliação de Algoritmos de Deteç̧ão de Espaços Espectrais Brancos para Aplicações de Rádio Cognitivo. Rio de Janeiro, 2014. 129p. Dissertação de Mestrado - Departamento de Engenharia Elétrico, Pontifícia Universidade Católica do Rio de Janeiro.

Com o desenvolvimento tecnológico no setor de telecomunicações, o espectro radioelétrico está quase totalmente ocupado com um grande número de múltiplas atribuições para os muitos serviços sem fio de aplicação comercial e, também, não comercial, tais como defesa, controle de tráfego aéreo e exploração científica. $\mathrm{O}$ espectro eletromagnético é um recurso natural precioso e escasso, por isso, importantes esforços estão sendo direcionados para o desenvolvimento de rádios cognitivos, com capacidade de sensoriar o uso do espectro e utilizar frequências momentaneamente disponíveis de forma oportunista. $\mathrm{O}$ rastreamento e a utilização de intervalos espectrais, ou white spaces, através da tecnologia de rádios cognitivos, permitirá aumentar a eficiência de uso do espectro com a introdução de novos serviços de telecomunicações a serem explorados por usuários secundários, obrigados a não interferir ou a provocar interferência muito limitada nos usuários primários. O objetivo geral deste trabalho é avaliar os principais algoritmos de detecção dos intervalos espectrais (Detector de Energia, Detecção do Valor Absoluto de Covariância, Sensoriamento de Covariância Espectral) por meio de simulações com dados experimentais obtidos em campanhas de medições e testes em laboratório. Os algoritmos foram testados para avaliar o seu desempenho em termos de probabilidade de detecção dada uma probabilidade de falso alarme requerida, complexidade computacional e robustez quanto a relações sinal-a-ruído baixas. Os dados experimentais utilizados provêm de campanhas de medidas realizadas em ambiente urbano na faixa de $3.5 \mathrm{GHz}$.

\section{Palavras-chave}

Rádio cognitivo; intervalos espectrais; algoritmos de detecção; complexidade computacional. 


\section{Abstract}

Molina Silva, Marcelo; da Silva Mello, Luiz Alencar Reis. Evaluation of Detection Algorithms of Spectral White Spaces for Cognitive Radio Applications. Rio de Janeiro, 2014. 129p. MSc. Dissertation Departamento de Engenharia Elétrico, Pontifícia Universidade Católica do Rio de Janeiro.

With the technological development of the telecommunications industry, the radio spectrum is almost fully occupied with a large number of multiple assignments for wireless services for both commercial and non-commercial applications, such as defense, air traffic control and scientific exploration. The electromagnetic spectrum is a precious and scarce natural resource. Therefore, a considerable effort is being directed at the development of cognitive radios, capable of sensoring the spectrum and using momentarily available frequency bands in an opportunistic way. The tracking and using of these spectral intervals, or white spaces, using cognitive radio technology will enhance the efficiency of the spectrum use and allow the introduction of new telecommunications services to be exploited by secondary users, obliged not to interfere or produce very limited interference to primary users. The aim of this study is to evaluate the main algorithms for detection of spectral intervals (Energy Detector, Detection of Covariance Absolute Value, Spectral Covariance Sensing) through simulations with experimental data obtained in field measurements campaigns. The algorithms were tested to evaluate their performance in terms of detection probability given a required false alarm probability, computational complexity and robustness in low signal-to-noise conditions. The experimental data used comes from the measurements campaigns in urban environments at the $3.5 \mathrm{GHz}$ band.

\section{Keywords}

Cognitive radio; spectral intervals; detection algorithms; computational complexity. 


\section{Sumário}

1 Introdução 14

1.1. Espectro de radiofrequências 16

1.2. Alocação do espectro radioelétrico para comunicações sem fio 17

1.3. Rádio cognitivo e sensoriamento do espectro 19

1.4. Objetivos 20

$\begin{array}{ll}\text { 1.5. Organização do Trabalho } & 21\end{array}$

2 Rádio Cognitivo 22

2.1. Mobilidade espectral. 24

2.2. Identificação de Localização 25

2.3. Acesso dinâmico e Descoberta de Rede / Sistema 25

2.4. Modulação / Codificação Adaptativa 26

2.5. Controle de Potência de Transmissão 26

2.6. Compartilhamento do Espectro 26

2.7. Sensoriamento do Espectro 28

2.8. Arquitetura da Rede de Rádio Cognitivo 29

2.8.1. Arquitetura de Rede 29

2.8.2. Sistema Primário e Sistema de Rádio Cognitivo 33

$\begin{array}{ll}\text { 2.8.3. Links em CRNs } & 34\end{array}$

3 Sensoriamento de Espectro 37

3.1. Desafios no sensoriamento do espectro 43

3.1.1. Requisitos de Hardware 43

3.1.2. Problema do Terminal Escondido 44

3.1.3. Espalhamento de Espectro 45

3.1.4. Duração entre Intervalos em Sensoriamentos 47

3.2. Métricas de Desempenho para o Sensoriamento Espectral. 47

3.2.1. Detecção de Energia (ED - Energy Detector) 49

3.2.2. Valor absoluto de covariância (CAV) 54

3.2.3. Sensoriamento Espectral de Covariância (SCS) 57 
4 Simulações $\quad 61$

4.1. Complexidade Computacional dos Algoritmos 61

4.1.1. Complexidade Computacional do Algoritmo de Detecção de Energia

4.1.2. Complexidade Computacional do Algoritmo de Valor absoluto de Covariância (CAV).

62

4.1.3. Complexidade Computacional do Algoritmo de Sensoriamento Espectral de Covariância (SCS).

4.1.4. Comparação da Complexidade Computacional dos diferentes Algoritmos de detecção. 68

4.2. Metodologia para a comparação das técnicas de detecção $\quad 70$

4.2.1. Dados Experimentais 71

4.2.2. Programa Synchromed 74

4.2.3. Resultados dos algoritmos de detecção. 78

4.2.4. Resultados da simulação do SCS para sinais individuais. 81

4.2.5. Efeito da probabilidade de falso alarme 84

5 Conclusões $\quad 89$

90

5.2. Sugestões para trabalhos futuros 91

6 Referências bibliográficas $\quad 92$

A Modelo Analítico e Estatístico. 99

A.1. Para o Detector de Energia (ED) 99

A.2. Para o Detector do valor absoluto de covariância (CAV) 104

B Rádio Definido por Software (SDR - Software Defined Radio) 105

B.1. GNU Radio 107

B.2. Universal Software Radio Peripheral (USRP) 111

B.3. Resultados obtidos na simulação usando a USRP. 112

B.4. Criação de um novo bloco tipo sync. 118

$\begin{array}{ll}\text { C Tempo de execução dos algoritmos. } & 127\end{array}$ 


\section{Lista de figuras}

Figura 1.1: Evolução do número de acessos. 15

Figura 1.2: Alocações de espectro no Brasil. 18

Figura 2.1: Propriedades do Rádio Cognitivo. 23

Figura 2.2: Vetor de alocação. 28

Figura 2.3: Rede Infraestruturada. 30

Figura 2.4: Arquitetura Ad-hoc. 31

Figura 2.5: Arquitetura de malha. 32

Figura 2.6: Links em CRNs.

Figura 3.1: Mecanismo de sensoriamento reativo. 40

Figura 3.2: Mecanismo de sensoriamento proativo. 41

Figura 3.3: Problema do terminal escondido. 45

Figura 3.4: Divisão do espectro realizado pelo espalhamento FHSS e os saltos de frequência. 46

Figura 3.5: Sinal convencional e DSSS 46

Figura 3.6: Energia recebida comparada com um limiar, com baixa potência de ruído. $\quad 49$

Figura 3.7: Exemplo de um sinal OFDM capturado com alta potência de ruído: caso detector de energia (ED). 50

Figura 3.8: Exemplo de um sinal OFDM capturado com alta potência de ruído: caso - Valor Absoluto de Covariância (CAV).

Figura 4.1: Complexidade Computacional dos Algoritmos:

ED, CAV, SCS, para Adições.

Figura 4.2: Complexidade Computacional dos Algoritmos:

ED, CAV, SCS, para Multiplicações. 69

Figura 4.3: Área de serviço usuário primário.

Figura 4.4: Símbolos OFDM. $\quad 72$

Figura 4.5: Campanha de medições com uma banda de 7 [MHz] com frequência central de $3.5[\mathrm{GHz}]$. 73

Figura 4.6: Amostra do sinal OFDM tomada a matriz da campanha de medições pelo programa Synchromed. 
Figura 4.7: No intervalo de início-fim estão as amostras do sinal OFDM, no intervalo de fim-início o ruído.

Figura 4.8: Seleção das amostras do sinal e ruído utilizando Syncromed. 77

Figura 4.9: Probabilidade de detecção em uma banda de 7 [MHz]. $\quad 78$

Figura 4.10: Probabilidade de detecção em uma banda de $3.5[\mathrm{MHz}] . \quad 79$

Figura 4.11: Probabilidade de detecção em uma banda de $1.7[\mathrm{MHz}] . \quad 80$

Figura 4.12: Probabilidade de detecção para sete grupo de sinais. 82

Figura 4.13: Sete grupos de sinais. 83

Figura 4.14: Detector de Energia (ED), Banda de 7 [MHz], $P f=5,10,15.84$

Figura 4.15: Valor Absoluto de Covariância (CAV), Banda de 7 [MHz], $\mathrm{Pf}=5,10,15$.

Figura 4.16: Sensoriamento de Covariância Espectral (SCS),

Banda de 7 [MHz], $\mathrm{Pf}=5,10,15$.

Figura 4.17: Detector de Energia (ED), Banda de 3.5 [MHz],

$\mathrm{Pf}=5,10,15$.

Figura 4.18: Valor Absoluto de Covariância (CAV), Banda de 3.5 [MHz], $\mathrm{Pf}=5,10,15$.

Figura 4.19: Sensoriamento de Covariância Espectral (SCS),

Banda de $3.5[\mathrm{MHz}], \mathrm{Pf}=5,10,15$.

Figura 4.20: Detector de Energia (ED), Banda de 1.7 [MHz],

$\mathrm{Pf}=5,10,15$.

Figura 4.21: Valor Absoluto de Covariância (CAV),

Banda de $1.7[\mathrm{MHz}], \mathrm{Pf}=5,10,15$.

Figura 4.22: Sensoriamento de Covariância Espectral (SCS),

Banda de $1.7[\mathrm{MHz}], \mathrm{Pf}=5,10,15$.

Figura B.1: Componentes do Hardware. 105

Figura B.2: Arquitetura do SDR. 106

Figura B.3: Esquema do funcionamento da plataforma GNU Radio em conjunto com o Universal Software Radio Peripheral. 108

Figura B.4: Blocos de processamento do GNU Radio. 110

Figura B.5: Diagrama simplificado de blocos do USRP. 112

Figura B.6: Dial Ton (GNU-Radio). 114

Figura B.7: Diagrama para a transmissão. 114 
Figura B.8: Diagrama de Blocos (GNU-Radio) para a transmissão.

Figura B.9: Diagrama de Blocos na Recepção. 115

Figura B.10: Diagrama de Blocos (GNU-Radio) para a recepção. 116

Figura B.11: Sinal no domínio da Frequência (GNU-Radio). 116

Figura B.12: Sinal no domínio do Tempo (GNU-Radio). 117

Figura B.13: Ocupação da largura de banda (GNU-Radio). 117

Figura B.14: Tela do terminal. 118

Figura B.15: Resposta do Terminal. 119

Figura B.16: Lugar no diretório onde se encontra o file sync_prov.h (Descargaslgr-inmetrolincludelinmetro). 120

Figura B.17: Dentro do file sync_prov.h. 120

Figura B.18: Lugar no diretório onde se encontra o file sync_prov_impl.h (Descargas lgr-inmetrolib). 121

Figura B.19: Dentro do file sync_prov_impl.h.

Figura B.20: Lugar no diretório onde se encontra o file sync_prov 1_impl.cc (Descargasigr-inmetrolib). 122

Figura B.21: Dentro do file sync_prov 1_impl.cc. 122

Figura B.22: Dentro do file sync_prov 1_impl.cc. 123

Figura B.23: Lugar no diretório onde se encontra o file inmetro_sync_prov.xml (Descargaslgr-inmetrolgrc). 123

Figura B.24: Dentro do file inmetro_sync_prov.xml. 124

Figura B.25: Terminal, comando make. 125

Figura B.26: Terminal comando. 125

Figura B.27: GNU-Radio-Companion. $\quad 126$

Figura C.1: Porcentagem dos tempos de processamento para a banda de $1.75[\mathrm{MHz}] . \quad 128$

Figura C.2: Porcentagem dos tempos de processamento para a banda de $3.5[\mathrm{MHz}]$. 128

Figura C.3: Porcentagem dos tempos de processamento para a banda de $7[\mathrm{MHz}]$. 


\section{Lista de tabelas}

Tabela 1.1: Tipos de serviços para cada frequência.

Tabela 2.1: Possível Uni-direcional Link no CRN

Tabela 3.1: Possíveis decisões tomadas pelo mecanismo de detecção de usuários primários.

Tabela 4.1: Complexidade computacional da média para o detector de energia.

62

Tabela 4.2: Complexidade computacional do desvio padrão para o detector de energia.

Tabela 4.3: Complexidade computacional para o cálculo das autocorrelações do valor absoluto de covariância (CAV).

Tabela 4.4: Complexidade computacional para a matriz covariância $\mathrm{C}$ do valor absoluto de covariância (CAV).

Tabela 4.5: Complexidade computacional para o teste estatístico do valor absoluto de covariância (CAV).

Tabela 4.6: Complexidade computacional para a obtenção da matriz $z(n)$ do sensoriamento espectrais de covariância (SCS).

Tabela 4.7: Complexidade computacional para a obtenção da matriz $M$ do sensoriamento espectral de covariância (SCS).

Tabela 4.8: Complexidade computacional para a matriz covariância $\mathrm{C}$ do sensoriamento espectral de covariância (SCS).

Tabela 4.9: Complexidade computacional para o teste estatístico do sensoriamento espectral de covariância (SCS).

Tabela 4.10: Complexidade computacional dos três algoritmos avaliados.

Tabela 4.11: Outras operações matemáticas $\quad 70$

Tabela C.1: Tempo de Rodagem dos programas ED, CAV, SCS 127 